\title{
GENERATION OF INTERNAL SOLITARY WAVE BY GRAVITY COLLAPSE
}

\author{
Chen-Yuan Chen \\ Department of Management Information System, Yung-Ta Institute of Technology and Commerce, Ping-Tung County, \\ Taiwan 90941, R.O.C. \\ John Rong-Chung Hsu \\ Department of Marine Environment and Engineering, National Sun YatSen University, Kaohsiung, Taiwan 80424, R.O.C. \\ NCKU-NSYSU Research Center for Ocean Environment and Technology, Taiwan; Honorary Research Fellow, School of \\ Civil and Resource Engineering, University of Western Australia, WA 6009, Australia.

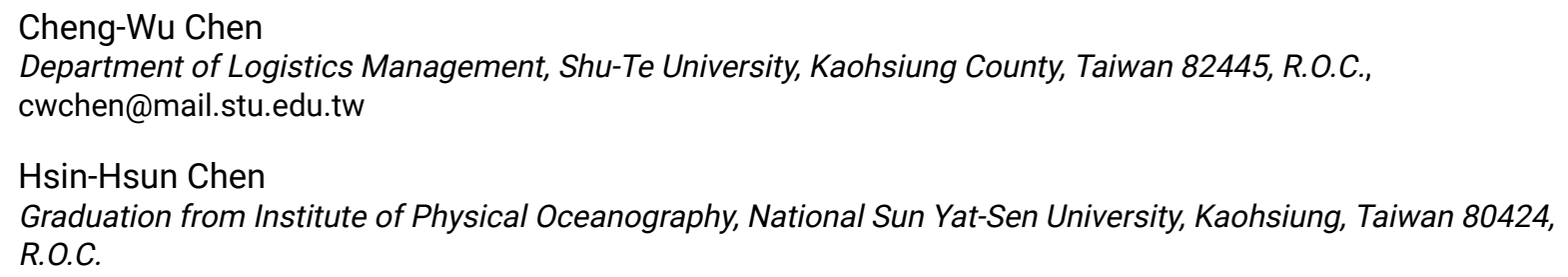

See next page for additional authors

Follow this and additional works at: https://jmstt.ntou.edu.tw/journal

Part of the Oceanography Commons

\section{Recommended Citation}

Chen, Chen-Yuan; Hsu, John Rong-Chung; Chen, Cheng-Wu; Chen, Hsin-Hsun; Kuo, Ching-Feng; and Cheng, Min-Hung (2007) "GENERATION OF INTERNAL SOLITARY WAVE BY GRAVITY COLLAPSE," Journal of Marine Science and Technology. Vol. 15: Iss. 1, Article 1.

DOI: $10.51400 / 2709-6998.2026$

Available at: https://jmstt.ntou.edu.tw/journal/vol15/iss1/1

This Research Article is brought to you for free and open access by Journal of Marine Science and Technology. It has been accepted for inclusion in Journal of Marine Science and Technology by an authorized editor of Journal of Marine Science and Technology. 


\section{GENERATION OF INTERNAL SOLITARY WAVE BY GRAVITY COLLAPSE}

\section{Acknowledgements}

The authors are grateful for the research grants to JRCH from the National Science Council, Republic of China under Grant No. NSC 90-2611-M-110-014 and NSC 91-2611-M-110-004. Thy wish to thank Dr. H.

Michallet, Laboratoire des Ecoulements Géophysiques et Industriels (LEGI), Institute National

Polytechnique de Grenoble, France for his assistance in the acquisition of the ultrasonic probes used in this study. Constructive suggestions from anonymous reviewers are also acknowledged.

\section{Authors}

Chen-Yuan Chen, John Rong-Chung Hsu, Cheng-Wu Chen, Hsin-Hsun Chen, Ching-Feng Kuo, and MinHung Cheng 


\title{
GENERATION OF INTERNAL SOLITARY WAVE BY GRAVITY COLLAPSE
}

\author{
Chen-Yuan Chen*, John Rong-Chung Hsu**,***, Cheng-Wu Chen****, \\ Hsin-Hsun Chen*****, Ching-Feng Kuo******, and Min-Hung Cheng**
}

Key words: gravity collapse, wave generation, internal solitary wave (ISW), depression-type ISW, elevation-type ISW.

\section{ABSTRACT}

Laboratory experiments were conducted to investigate the generation of an internal solitary wave (ISW) in a two-layer free surface fluid system in wave flume $(12 \mathrm{~m} \times 0.5 \mathrm{~m} \times 0.7 \mathrm{~m})$, which included wave channel and compartment. There is filled with stratified twolayer fluid system in the channel and small compartment, respectively. These two regions in the wave flume are separated by a movable vertical gate at one end of the flume for generating the ISW (internal solitary wave). An ISW generation is thus caused by gravity collapse upon raising the vertical gate. Given positive potential energies, an elevation-type ISW is followed by an anticlockwise overturning motion at the interface; on the other hand, an ISW of depression-type was generated by clockwise motion. This paper presented physical properties related to wave generation in a stratified fluid in laboratory. In the wave flume, the stable wave propagation, either elevation-type or depression-type ISW, is influenced by environmental condition. An ISW of depression transferred into unstable fluctuation instead of soliton feature whilst the upper layer thickness is greater than the lower layer thickness. Similarly, an ISW of elevation reduces its wave amplitude and causes trailing oscillation followed with the leading wave.

\section{INTRODUCTION}

Water waves play a key role in the ocean, especially those on the sea surface caused by the interaction

Paper Submitted 07/07/05, Accepted 01/06/06. Author for Correspondence: Cheng-WuChen.E-mail: cwchen@mail.stu.edu.tw.

*Department of Management Information System, Yung-Ta Institute of Technology and Commerce, Ping-Tung County, Taiwan 90941, R.O.C.

**Department of Marine Environment and Engineering, National Sun Yat-

Sen University, Kaohsiung, Taiwan 80424, R.O.C.

***NCKU-NSYSU Research Center for Ocean Environment and Technology,

Taiwan; Honorary Research Fellow, School of Civil and Resource Engineering, University of Western Australia, WA 6009, Australia.

****Department of Logistics Management, Shu-Te University, Kaohsiung County, Taiwan 82445, R.O.C.

*****Graduation from Institute of Physical Oceanography, National Sun Yat-Sen University, Kaohsiung, Taiwan 80424, R.O.C.

******Department of Color Filter Inspection, Chi Mei Optoelectronics, Tainan County, Taiwan, R.O.C. between the ocean and atmosphere. When a flat surface is displaced from an equilibrium position, it regains its position with a restoring force affected by both surface tension and the gravity force, respectively, with periods up to 20 seconds, wave speeds up to tens of meters per second, and amplitudes of up to several hundred meters approximately. However, waves can also occur between any two fluids or same fluid in different densities, and oceanographers refer to these waves occurring between ocean layers of different densities as internal waves which are more easily produced than the surface

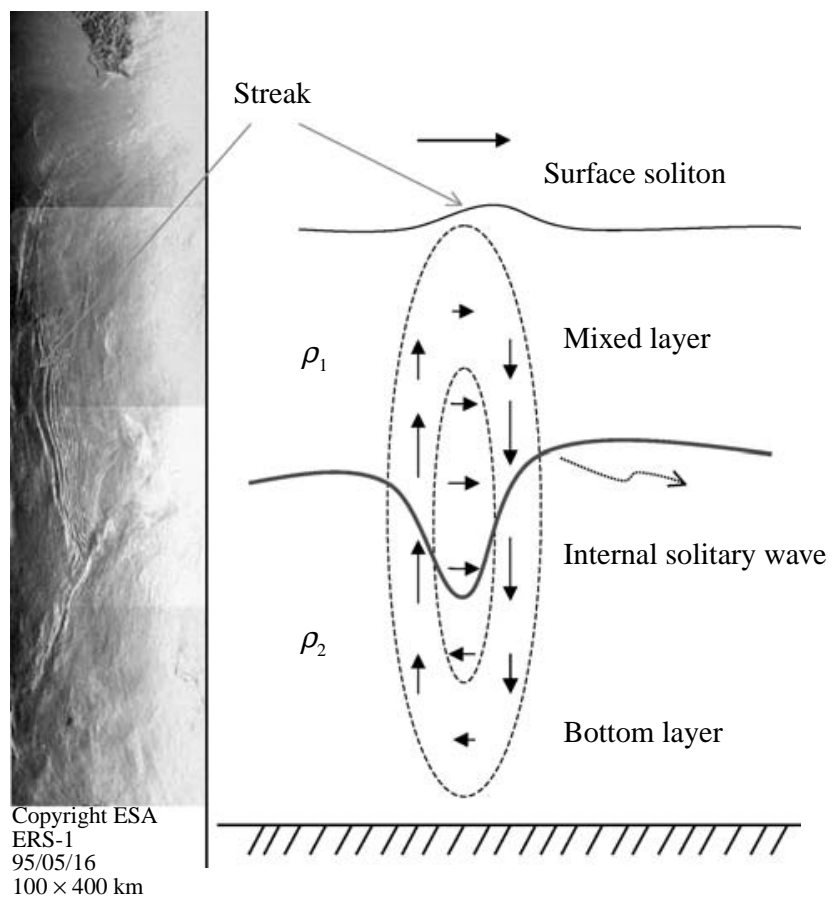

Fig. 1. Sketch of an internal wave propagating on the thermocline in the coastal ocean, and structure of streamlines causing surface streak contrast with a vertical view of SAR images. (a) A strip of 4 frames of ERS-1 SAR images $(100 \times 400 \mathrm{~km})$ collected south of Taiwan in Luzon Strait on June 16, 1995, showing huge internal soliton packet located near $120^{\circ} \mathrm{E}$, and $20.5^{\circ} \mathrm{N}$ (from [10]); (b) Thick solid line indicates internal wave of depression. The soliton on the surface are produced by the convergence of water above the wave troughs in the mixed layer (from [20]). 
waves. The effect of an internal wave may be visible on ocean surface, as seen in Figure 1, from the convergence of surface particle movement above its troughs [10, 15, 20]. The interface between two superposed fluids of different densities can similarly support gravity waves. The restoring forces resulting from relative buoyancy and gravity are weaker, produce typically periods with internal waves from minutes to hours, wavelengths from tens of meters to a few tens of kilometers, and amplitudes from few meters to several tens of meters $[18,19]$.

Opinions are widely divided for internal wave generation. For the internal waves in the ocean, they may be generated by a number of external forces, such as strong wind, tidal motion, ocean current and atmospheric pressure fluctuations. A great deal of reviews on detailed process portraying internal wave generation mechanisms had been presented by Garret and Munk [9], Thorpe [23], and LeBlond and Mysak [14]. Tidal current over a submarine topography also produces an internal wave field at the pycnocline between upper and lower layer fluid [1]. The mechanisms of internal wave generation in the East and South China Seas may include the influence of the tide and the upwelling, which is induced by the intrusion of the Kuroshio Current across the continental shelf, ridges or sills [10, 15]. Additional, atmospheric generation may be influenced by travelling pressure and stress fields and a variable buoyancy flux [23]. The resonant interaction of a pair of surface waves [8], instabilities in the mean current [18] and geostrophic adjustment after flow instabilities [13] are also likely sources of internal waves. In long narrow lakes, first baroclinic mode internal wave packets can be excited in response to strong, significant wind events [7, 22]. Mechanical energy from travelling strong winds is responsible for the generation of a large fraction of internal waves in the ocean at all depths, and interior mixing in the ocean is considered to be originally supplied at large scales by atmospheric forcing [13]. However, it is difficult, though not impossible, to thoroughly investigate the characteristics of wave gen- eration in field observations. Therefore, alternative approach using laboratory facilities to study nonlinear internal waves have become a viable means on long internal gravity waves [12]. Some studies considering wave properties in the laboratory are investigated by Segur and Hammack [21], Kao et al. [11], Michallet and Barthelemy [16, 17], and Chen et al. [2-4]. However, these works did not report the process of wave generation, except Kao et al. [11] who proposed the so-called "collapse mechanism". Even so, the development of an ISW from the wave source was not discussed in Kao et al. [11]. In the present study, we detail the development of an ISW, in both elevation and depression-types in the laboratory.

\section{EXPERIMENTAL SETUP}

Laboratory experiments were conducted in a steelframed wave flume of $12 \mathrm{~m}$ long, with a cross-section of $0.7 \mathrm{~m}$ high by $0.5 \mathrm{~m}$ wide supported by a polycarbonate bottom panel. The experimental apparatus are given schematically in Figure 2. A removable sluice gate panel for generating internal waves was mounted on the right hand side of the flume. No wave absorbing facilities were introduced at either end of the flume; thus incident waves were free to reflect back to the test section between the slope and the gate. Devices with rigid slope and impermeable bottom plane allowed an internal wave reflecting back and forth while propagating in the flume, hence wave attenuation by energy damping primarily originated from bottom friction took up more than several tens of minutes approximately.

The proposed laboratory experiments were performed in the wave flume with a two-layer fluid system of fresh and brine water. The upper layer was fresh water with density $\rho_{1}$, to a depth $H_{1}$. The fresh water body was allowed to stand overnight for the temperature to equilibrate with the ambient condition. The medium of the lower layer was brine water with density $\rho_{2}$, which was premixed separately in a constant head tank and filled slowly into the flume to a depth of $\mathrm{H}_{2}$ by

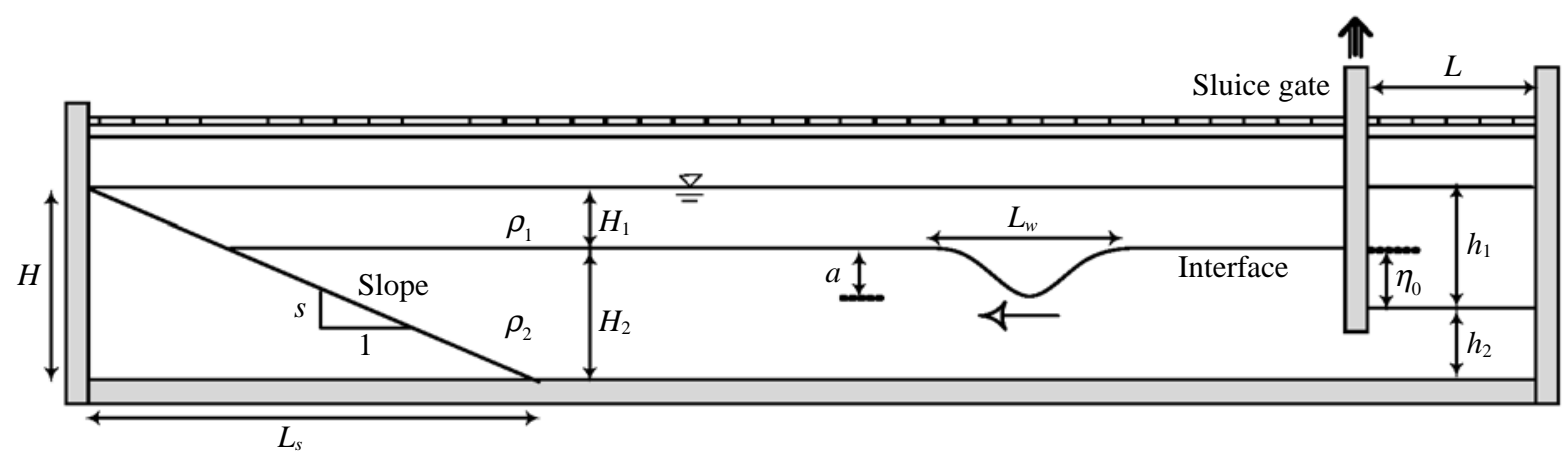

Fig. 2. A schematic view showing on experimental set-up for internal wave reflection from a uniform slope in a two-layer fluid system. 
gravity through several small openings along the bottom of the flume. Brine water had to be carefully infused beneath the fresh water, with a thin piece of sponge placed at every entry point to ensure uniform diffusion into the two-layer fluid system with minimum disturbance and mixing at the interface. The filling time depended on the upper and lower layer thickness. For example, with discharge about $600 \mathrm{~cm}^{3} / \mathrm{sec}$ for the fresh water and $160 \mathrm{~cm}^{3} / \mathrm{sec}$ for the brine water, it would take about two hours to produce a two layered system of $H_{1} /$ $\mathrm{H}_{2}=30 / 10$ (units in centimeters). Colouring agent was injected afterwards through a set of injector at the interface of the fluid system, thus producing a visible sharp interface between limpid fluids with the brine water beneath the fresh water. Densities of water for the upper layer were maintained at $999 \mathrm{~kg} / \mathrm{m}^{3}$ and about $1030 \mathrm{~kg} / \mathrm{m}^{3}$ approximately for the lower layer, producing stratified fluids environment of Boussinesq parameter $\sigma \cong 0.03$. The Boussinesq parameter represents the ratio of density difference and reference density, and the value comes to around 0.03 in the ocean.

Internal solitary waves were generated by overturning the interfacial water behind the movable gate. The mechanism to generate either a single solitary wave or a train of solitary waves could be estimated [11]. First, a mini pump was used to remove a small quantity of fresh water from the main section of the flume to the small compartment behind the vertical gate. A corresponding mass of the brine water then slowly moved to the other side of the gate through a narrow gap between the bottom of the gate and the flume bottom, such maintaining a hydrostatic balance. This created a prescribed step difference $£$ bo in the interface levels on either side of the gate. By raising the movable gate using a pneumatic device controlled by a switch, an initial internal wave was generated by overturning the brine water, causing a leading solitary wave to propagate in the flume. A primary internal wave developed into a leading solitary wave propagating ahead of a transient dispersive wave train. Once the thickness of

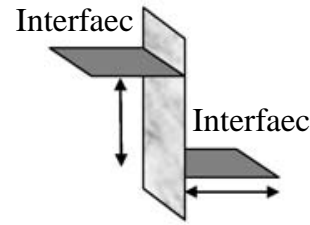

(a)

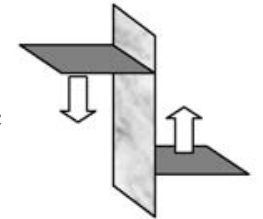

(b)

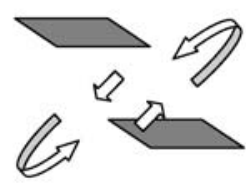

(c)
Fig. 3. Illustration of the sequence of a gravity collapse event following the lifting of a gate; (a) a step depth $\eta_{0}$ showing difference in interface level either side of the movable gate; (b) a restoring force by which gravity force of the LHS and buoyancy force of the RHS were evoked following the lifting of the gate, and (c) an overturning potential causing vortex motion. fresh water in the small compartment was greater than that in main section of the flume, clockwise overturning on the interface level grew while raising the gate, or vice versa. The evolution of a depression-type internal wave resulted due to clockwise interface overturning; on the contrary, elevation-type internal wave could be generated by counterclockwise interface overturning. In order to minimise the attenuation of pycnocline diffusion, test runs were first carried out with small amplituded waves to minimize mixing at the interface, then progressively with large amplituded waves. In this way, the interface was able to satisfactorily support about 56 test runs before having to drain and refill the flume later.

\section{OBSERVATION ON ISW GENERATION}

Internal waves were generated through a collapse mechanism from a difference in potential on either side of the removable gate. It has been well known that the number of solitons generated in a wave flume can be pre-determined by a method given by Kao et al. [11] who experimentally investigated the evolution of the disturbance as a function of the upper and lower layer fluid thickness, the step length (i.e. distance from the gate to the upstream end of the flume; $L$ in Figure 2) and step depth (i.e. difference in the interface levels on either side of the removable gate; $\eta_{0}$ in Figure 2 ). The internal waveforms were also influenced by fresh/brine water head on either side of the gate used in this study. Kao et al. [11] has given an empirical equation for the number of solitons $N$ as:

$$
N \leq \frac{L}{\pi} \sqrt{\frac{3}{2}\left|\frac{H_{1}-H_{2}}{H_{1}^{2} H_{2}^{2}}\right| \eta_{0}}+1
$$

where $H_{1}$ and $H_{2}$ are thickness of the upper and lower layer fluid, respectively (see Figure 2), $L$ the step length, $\eta_{0}$ the step depth (see Figures 2 and 3). The step length $L$, being the width of the minor water compartment, was kept constant at $0.3 \mathrm{~m}$ distance in all experiments. Thereby, by controlling the stratification depth ratio $H_{1} /$ $H_{2}$ and the step depth $\eta_{o}$ for the difference in interface levels separated by the gate, any number of solitons required could be generated. According to Eq. (1) and for the experimental configuration adopted in this study, the number of solitons expected is summarized in Table 1. The values given in Table 1 represent an upper bound, i.e. the actual number of solitons generated was less than or equal to the value given. In reality, the estimated values may be rounded down to give an integer given in the brackets.

Upon lifting the vertical gate pneumatically, local 
vortices formed immediately within the proximity of the gate. Consequently, ISWs developed due to an interfacial imbalance of the head difference on either side of the gate, i.e. the step depth $\eta_{o}$ which limited the maximum "potential" amplitude of the wave (Figure 3 ). In order to trace the movement of the interface between the two fluids, a patch of dye was transfused at different depths of interface both in front of and behind the gate. A schematic diagram showing the direction of overturning motion in the vicinity of wave source is given in Figure 3. The action leads to an ISW of depressiontype. An event history of successive development in Figure 4 shows an overturning motion, similar to a vortex, was generated. Fresh water in the small compartment produced an internal bore while raising the vertical gate, seen in Figure 4c. The internal bore then evolved a depression-type wave and propagated ahead in the main section of the flume, shown in Figure 4d, due to potential energy supplied by beginning interface difference. Better depression ISW feature was apparent with time after 2-4 seconds (Figures $4 \mathrm{e}$ and $4 \mathrm{f}$ ). Tracer attenuation was produced, seen in Figures $4 \mathrm{~g}$ and $4 \mathrm{~h}$, due to mixing between the upper and lower fluid in the process of wave generation. Those indicated that the vortex was in a clockwise rotation leading to an ISW of depression-type, compared to an anticlockwise direction leading to an ISW of elevation-type in Figure 5 when the head of brine water behind the gate was higher than that in the main section of the flume.

\section{DISCUSSION ON EXPERIMENTAL RESULTS}

Shear instabilities in a stratified two-fluid system caused by a relative motion in opposite direction induced mixing at the source of wave motion [6]. A developing wave form then propagated towards the main section of the flume with a high degree of mixing at its rear end. The mixing was more pronounced for large step depth $\eta_{o}$. The rear end of the developing wave sharpened with no sign of mixing once it had propagated approximately $1.5 \mathrm{~m}$ from the source. The dissipation of energy was mainly due to turbulent mixing, frictional losses and viscous effect, thus the amplitude of an internal wave in the main section of constant depth in the flume was less than the potential difference $\eta_{o}$. As

Table 1. Prediction of the number of solitons for each experiment

\begin{tabular}{cccc}
\hline Configuration & $\eta_{o}=5 \mathrm{~cm}$ & $\eta_{o}=10 \mathrm{~cm}$ & $\eta_{o}=20 \mathrm{~cm}$ \\
\hline$H_{1}=10 \mathrm{~cm} ; H_{2}=30 \mathrm{~cm}$ & $1.37[1]$ & $1.53[1]$ & $1.75[1]$ \\
$H_{1}=30 \mathrm{~cm} ; H_{2}=10 \mathrm{~cm}$ & $1.37[1]$ & $1.53[1]$ & $1.75[1]$ \\
$H_{1}=35 \mathrm{~cm} ; H_{2}=5 \mathrm{~cm}$ & $1.79[1]$ & $2.12[5]$ & $2.58[5]$ \\
\hline
\end{tabular}

discussed earlier, the process could at times lead to the production of a second solitary wave with much smaller amplitude than the first one and propagated at much slower speed. This second wave was found to interact with the main solitary wave reflected from the slope without causing additional mixing or breaking in the stratified fluids. The two progressive ISWs were observed to pass each another seemingly without change in waveform and properties. In addition, lifting of the gate also produced minor perturbations at the air-water interface. This was due to the abrupt nature of the gate lifting plus droplets of water falling from the gate onto the water surface. The effect of lifting the gate was similar to an accelerating mass on a fabric as the ripple on a pond disturbed by a rock. The oscillation of the barotropic mode in a seiche-like fashion suggested that other free surface had been agitated; however, this high frequency signal was quickly damped within a matter of seconds.

In the small compartment behind the gate, interface level was different from the main section for wave
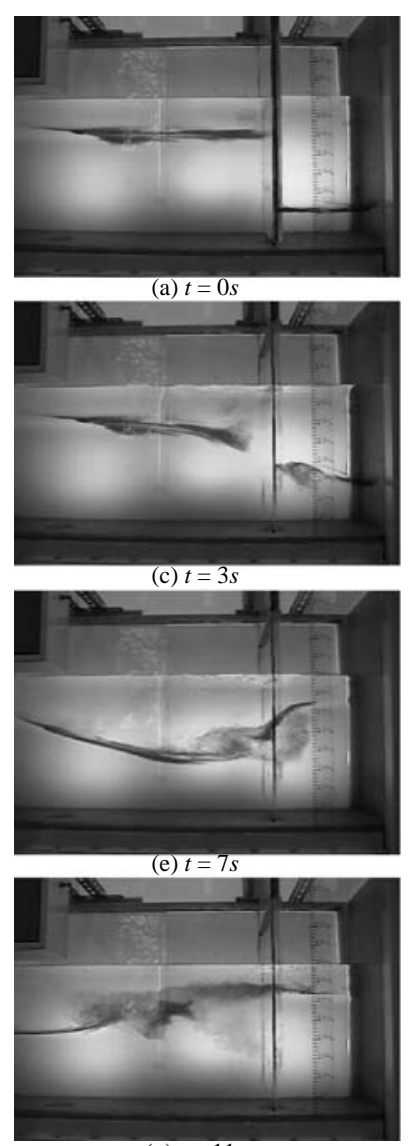

(g) $t=11 \mathrm{~s}$

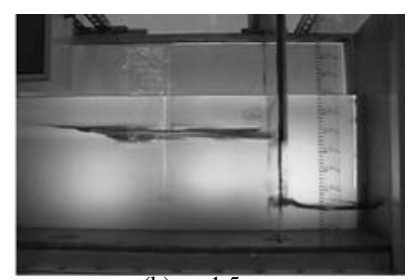

(b) $t=1.5 \mathrm{~s}$
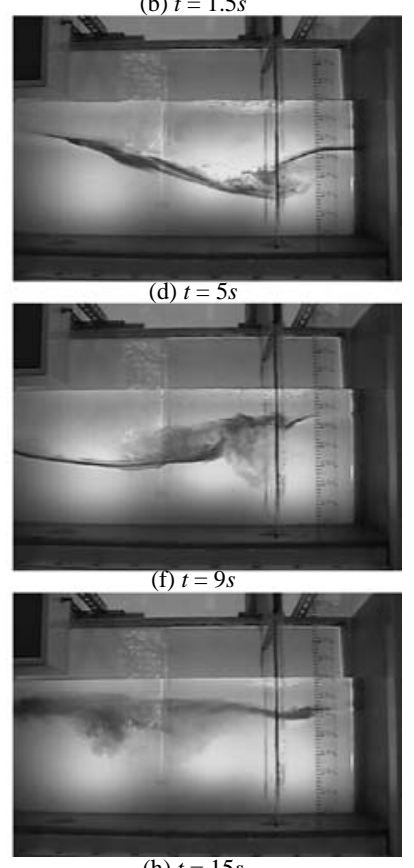

(h) $t=15 \mathrm{~s}$
Fig. 4. Video imagery of successive events indicating a clockwise overturning motion leading to the generation of an ISW of depression-type for experimental condition of $H_{1}=10 \mathrm{~cm}$, $H_{2}=30 \mathrm{~cm}, h_{1}=30 \mathrm{~cm}$, and $h_{2}=10 \mathrm{~cm}$. 
propagation on the other side of the gate, due to a small quantity of fresh water was removed or added to the small compartment. As a result, gravitational potential energy was produced by a difference in the interface levels separated by the vertical gate. If the fluid system maintained sufficient potential by the time when a step depth reached its scheduled condition, lifting of the gate would partially converted it to kinetic energy, thus developing a solitary wave forward into the main section. In the process of energy conversion, fragmental energy losses in frictional and viscous effect were indispensable. However, it was apparent that internal waveforms were subjected to rotation (clockwise or anticlockwise) of overturning of water mass associated with the generation of an ISW of depression or elevation type. As seen in Figure 6, a controlled condition of $h_{1}>H_{1}$ (i.e. $h_{1}$ is the fluid thickness of the upper layer in the small compartment) would supply fresh water with an elevation head to develop a clockwise overturning motion causing an ISW of depression type; alternatively, for $h_{2}$

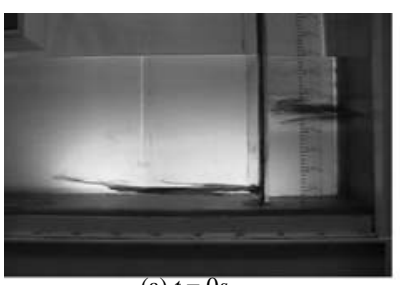

(a) $t=0 s$

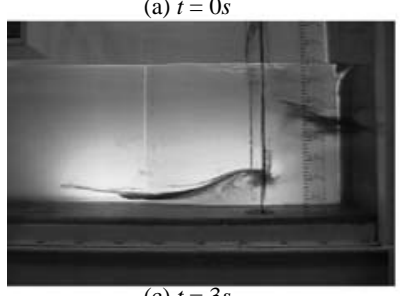

(c) $t=3 \mathrm{~s}$

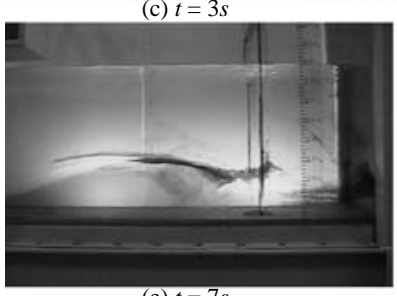

(e) $t=7 \mathrm{~s}$

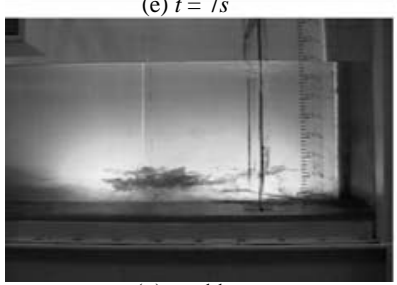

(g) $t=11 \mathrm{~s}$

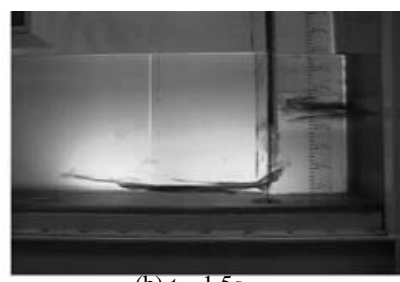

(b) $t=1.5 \mathrm{~s}$

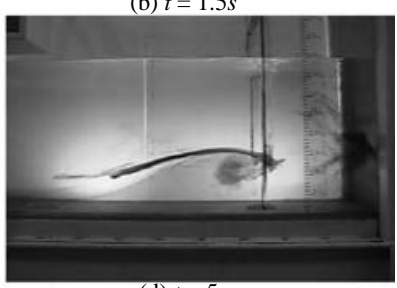

(d) $t=5 \mathrm{~s}$

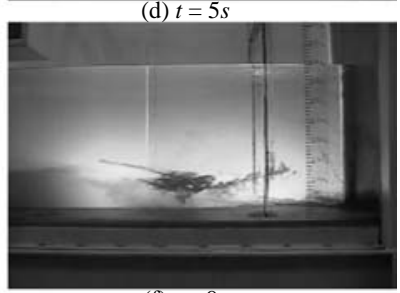

(f) $t=9 \mathrm{~s}$

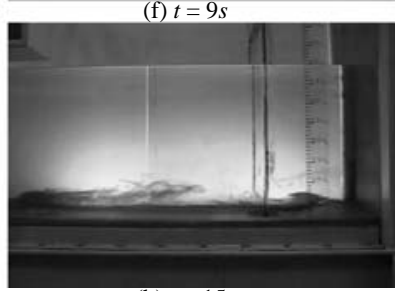

(h) $t=15 \mathrm{~s}$
Fig. 5. Video imagery of successive events indicating an anticlockwise overturning motion leading to the generation of an ISW of elevation-type for experimental condition of $H_{1}=30 \mathrm{~cm}$, $H_{2}=10 \mathrm{~cm}, h_{1}=10 \mathrm{~cm}$, and $h_{2}=30 \mathrm{~cm}$.
$>\mathrm{H}_{2}$, a higher brine water head in the small compartment would develop an ISW of elevation type. The phenomena described above are also illustrated in the subsequent events of Figures 4 and 5, showing developments in consecutive time. The brine elevation head in the small compartment represented potential energy of a two-layer fluid system relative to that in the main section. In the experiments performed, however, stable elevation/depression wave propagation in the main section of the flume could maintain only if depth ratio $H_{1} /$ $H_{2}$ was set correctly in the main compartment. Here the so-called environmental condition $H_{1} / H_{2}$ referred to the relative magnitude of either $H_{1}>H_{2}$ or $H_{1}<H_{2}$. Even if a depression-type wave could be generated at wave source, it might not maintain a stable form while propagating in the main section of the flume, unless the condition of $H_{1} / H_{2}$ was environmentally suitable.

Four classifications for successfully generating
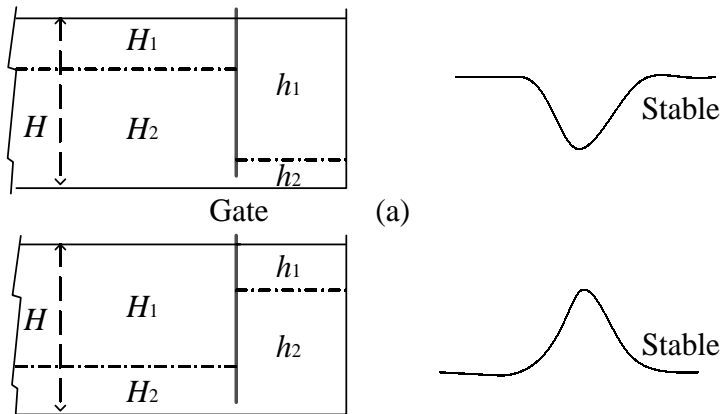

(a)

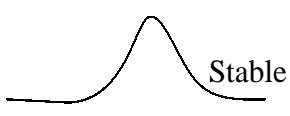

(b)
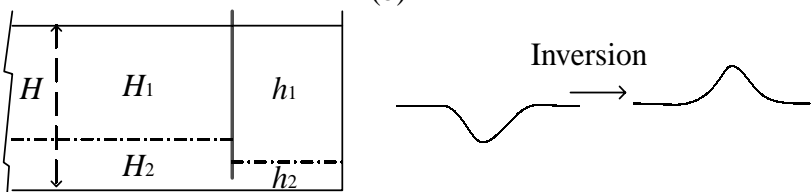

(c)

\begin{tabular}{|c|c|c|}
\hline & $H_{1}$ & - \\
\hline & $H_{2}$ & $h_{2}$ \\
\hline
\end{tabular}

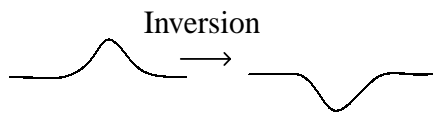

(d)

Fig. 6. Laboratory arrangements for producing stable and unstable wave form of internal waves in a two-layered fluid system. Four combinations of water levels were arranged for fluid thickness ratio of fresh and brine fluid on either side of a vertically removable gate. The variations in water levels on the LHS of each sub-graph are configuration adopted in this study, while the RHS gives waveform development in the main section of the wave flume following the lifting of the gate. Configuration in part (a) stable IGW of depression-type; (b) stable IGW of elevation-type; (c) an unstable form of depression and later inverted into an ill-formed IGW of elevation; and (d) an unstable IGW of elevation and later developed into an illformed IGW of depression. 
internal waves in the wave flume are illustrated in Figure 6. For example, under the arrangement of $h_{1}>H_{1}$ and $H_{1}>H_{2}$, original depression-type ISW would gradually evolve into an "elevation" type internal wave. This unstable phenomenon of wave inversion was supported by wave profiles as given in Figure 7, which described the time series of the internal wave. The wave profiles are collected by an ultrasonic probe and processed by control software, Multi Nodes Data Acquisition System (MNDAS). These manipulations were reported in details by Chen et al. [5]. As a result, the generation of stable soliton feature in a wave flume was satisfied by a strict configuration including the potential difference and corresponding environmental condition, as seen in Figure 8; otherwise, an ISW form would be inverted later and became unstable non-periodic oscillation.

\section{SUMMARY}

Stable waveform for either elevation or depression-type ISW in the laboratory agrees well with the field observation by Liu et al. [15] in the South China Sea. Stable propagation is governed by an appropriate depth factor, which is called "environmental condition" in this study. However, a depression-type ISW could not inverse into elevation-type since the upper layer thickness was greater than the lower one in the wave flume. Equivalently, an elevated ISW could not inverse into a depression ISW while the lower layer thickness was greater than the upper one. Wave profiles obtained by experimental results illustrated that perfect inver-

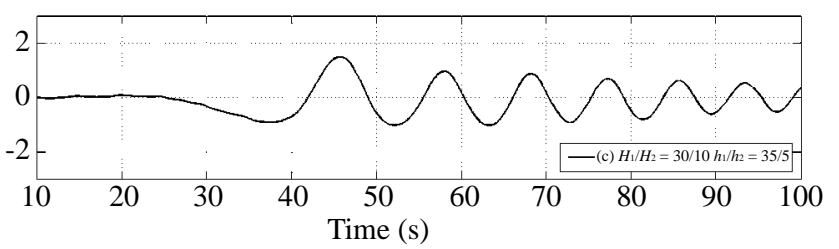

Fig. 7. Recorded wave profiles of a depression-type ISW followed by non-periodic trailing oscillations in experimental configurations of $h_{1}>H_{1}$, and $H_{1}>H_{2}$. The $x$-axis is time in second, and $y$-axis is the wave height in centimeter.

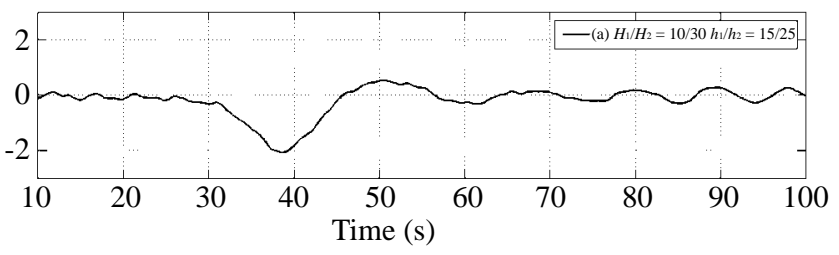

Fig. 8. Recorded wave profiles of a perfect depression-type ISW produced by experimental configurations of $h_{1}>H_{1}$, and $H_{1}<H_{2}$. The $x$-axis is time in second, and $y$-axis is the wave height in centimeter. sion for soliton feature between ISWs of elevation and depression type in the wave flume is not permitted. Most of oceanographers speculated that perfect inversion from a depression to an elevated type would appear while an ISW over transition zone due to the change of the environmental condition (i.e. $H_{1}<H_{2} \rightarrow H_{1}>H_{2}$ ); however, the evidence have not been discovered by field experiments. According to the experimental results in the laboratory, we strongly suggest that a depression ISW would decay on the continental shelf, in which the upper layer is thicker than the lower layer (i.e. environmental condition of $H_{1}>H_{2}$ ), and an elevated ISW would be enhanced at the back of the leading depression ISW. Hypothetical, the maturation of an elevated ISW resulted from energy transformation by the decaying depression ISW.

\section{CONCLUSION}

1. According to Eq. (1), the number of solitons to be generated for this study was kept to one in the wave flume. The estimated value is useful to generate a perfect solitary waveform in a stratified two-layer fluid system.

2. By means of video imagery of successive events during laboratory experiments, it indicates that if the upper layer is thinner than the lower layer (i.e. environmental condition of $H_{1}<H_{2}$ ), internal wave of depression may be generated; conversely, internal waves of elevation type yield when $H_{1}>H_{2}$.

3. Generated by the mechanism of gravity collapse, a propagating ISW in the wave flume maintain its soliton feature if the environmental condition permitted. Otherwise, a train of wave oscillations occurs following after the leading wave of the ISW.

\section{ACKNOWLEDGEMENTS}

The authors are grateful for the research grants to JRCH from the National Science Council, Republic of China under Grant No. NSC 90-2611-M-110-014 and NSC 91-2611-M-110-004. Thy wish to thank Dr. H. Michallet, Laboratoire des Ecoulements Géophysiques et Industriels (LEGI), Institute National Polytechnique de Grenoble, France for his assistance in the acquisition of the ultrasonic probes used in this study. Constructive suggestions from anonymous reviewers are also acknowledged.

\section{REFERENCES}

1. Bell, Jr. T.H., "Topographically Generated Internal Waves in the Open Ocean," Journal of Geophysical Research, Vol. 80, pp. 320-327 (1975). 
2. Chen, C.Y., Hsu, J.R.C., Chen, H.H., Kuo, C.F., and Cheng, M.H., "Laboratory Observations on Internal Solitary Wave Evolution on Steep and inverse Uniform Slopes," Ocean Engineering, Vol. 34, pp. 157-170 (2007).

3. Chen, C.Y., Hsu, J.R.C., Cheng, M.H., Chen, H.H., and Kuo, C.F., "An Investigation on Internal Solitary Waves in a Two-Layer Fluid: Propagation and Reflection from Steep Slopes," Ocean Engineering, Vol. 34, pp. 171-184 (2007).

4. Chen, C.Y., Hsu, J.R.C., Kuo, C.F., Chen, H.H., and Cheng, M.H., "Laboratory Observations on Internal Wave Evolution Over a Submarine Ridge," China Ocean Engineering, Vol. 20, pp. 61-72 (2006).

5. Chen, C.Y., Hsu, J.R.C., Kuo, C.F., Chen, H.H., and Cheng, M.H., "Wave Propagation at the Interface of a Two-Layer Fluid System in the Laboratory," Journal of Marine Science and Technology, Vol. 15, pp. 8-16 (2007).

6. Dunkerton, T.J., "Shear Instability of Internal Inertiagravity Waves," Journal of Atmospheric Sciences, Vol. 54, No. 12, pp. 1628-1641 (1997).

7. Farmer, D.M., "Observation of Long Nonlinear Internal Waves in a Lake," Journal of Physical Oceanography, Vol. 8, No. 1, pp. 63-73 (1978).

8. Garrett, C. and Munk, W., "Space-Time Scales of Internal Waves," Geophysical fluid Dynamics, Vol. 3, pp. 225-264 (1972).

9. Garrett, C. and Munk, W., "Internal Waves in the Ocean," Annual Review of Fluid Mechanics, Vol. 11, pp. 339-369 (1979).

10. Hsu, M.K., Liu, A.K., and Liu, C., "A Study of Internal Waves in the China Sea and Yellow Sea using SAR," Continental Shelf Research, Vol. 20, pp. 389-410 (2000).

11. Kao, T.W., Pan, F.S., and Renouard, D., "Internal Solitions on the Pycnocline: Generation, Propagation, Shoaling and Breaking Over a Slope," Journal of Fluid Mechanics, Vol. 159, pp. 19-53 (1985).

12. Koop, C.G. and Butler, G., "An Investigation of Internal Solitary Waves in a Two-Fluid System," Journal of
Fluid Mechanics, Vol. 112, pp. 225-251 (1981).

13. Kundu, P.K., "On Internal Waves Generated by Travelling Wind," Journal of Fluid Mechanics, Vol. 254, pp. 529-559 (1993).

14. LeBlond, P.H. and Mysak, L.A., Waves in the Ocean, Elsevier Scientific, Great Britain (1978).

15. Liu, A.K., Chang, Y.S., Hsu, M.K., and Liang, N.K., "Evolution of Nonlinear Internal Waves in East and South China Seas," Journal of Geophysical Research, Vol. 103, pp. 7995-8008 (1998).

16. Michallet, H. and Barthelemy, E., "Ultrasonic Probes and Data Processing to Study Interfacial Solitary Waves," Experiments in Fluid, Vol. 22, pp. 380-386 (1997).

17. Michallet, H. and Barthelemy, E., "Experimental Study of Large Interfacial Solitary Waves," Journal of Fluid Mechanics, Vol. 366, pp. 159-177 (1998).

18. Muller, P., Holloway, G., Henyey, F., and Pomphrey, N., "Nonlinear Interactions Among Internal Gravity Waves," Reviews of Geophysics, Vol. 24, No. 3, pp. 493-536 (1986).

19. Olbers, D.J., "Models of the Oceanic internal Wave Field," Reviews Geophysics and Space Physics, Vol. 21, pp. 1657-1606 (1983).

20. Osborne, A.R. and Burch, T.L., "Internal Solitons in the Andaman Sea," Science, Vol. 208, No. 4443, pp. 451460 (1980).

21. Segur, H. and Hammack, J.L., "Soliton Models of Long internal Waves," Journal of Fluid Mechanics, Vol. 118, pp. 285-304 (1982).

22. Stevens, C., Lawrence, G., Hamblin, P., and Carmack, E., "Wind Forcing of Internal Waves in a Long Narrow Stratified Lake," Dynamics of Atmospheres and Oceans, Vol. 24, pp. 41-50 (1996).

23. Thorpe, S.A., "The Excitation, Dissipation, and Interaction of Internal Waves in the Deep Ocean," Journal of Geophysical Research, Vol. 80, No. 3, pp. 328-338 (1975). 